\title{
On the effects of new reaction rates on proton-capture nucleosynthesis in low mass stars
}

\section{Sara Palmerini*}

Dipartimento di Fisica Università degli Studi di Perugia and INFN sezione di Perugia, Italy

E-mail: sara.palmeriniefisica.unipg.it

\section{Mauizio Busso}

Dipartimento di Fisica Università degli Studi di Perugia and INFN sezione di Perugia, Italy

\section{Marco La Cognata}

Dipartimento di Metodologie Fisiche e Chimiche per l'Ingegneria, Università di Catania and Laboratori Nazionali del Sud - INFN, Catania, Italy

\section{Sergio Cristallo}

Departamento de Fisica Teorica y del Cosmos, Universidad de Granada, Spain and Osservatorio di Teramo, INAF, Italy

Evolved low- and intermediate-mass stars are important sites for the production of heavy nuclei (through slow n-captures) and of light- and intermediate-mass isotopes (through p-captures). This was revealed by spectroscopy of evolved stars as well as by the chemical analysis of presolar grains of circumstellar origins. The observational basis for these issues has grown considerably over the years, confirming peculiar abundances of ${ }^{3} \mathrm{He},{ }^{7} \mathrm{Li}, \mathrm{CNO}$ isotopes and ${ }^{26} \mathrm{Al}$. Many studies have been presented in the last thirty years suggesting that the above evidence can be accounted for by non-convective transport mechanisms that link the stellar envelope with the region where pcaptures take place, during the red giant phases. Nucleosynthesis in deep mixing episodes is made uncertain by two series of problems. On one side one has to identify the physical mechanism for the transport. On the other, several reaction rates adopted in the calculations are still uncertain, mainly because hydrogen burning takes place in red giants at typical Gamov-Peak energies of a few tens of $\mathrm{keV}$, where experimental measurements are extremely difficult. We show how the use of recent and accurate reaction rates induces considerable differences in the nucleosinthesis results as compared to previous studies. Among the most remarkable novelties we discuss the effects of the new rates for the ${ }^{14} \mathrm{~N}(\mathrm{p}, \gamma){ }^{15} \mathrm{O}$ and for $\mathrm{p}$-captures on oxygen isotopes. They jointly provide a much better agreement than before with presolar grain isotopic abundances and allow for a clear determination of the mass of the parent star.

11th Symposium on Nuclei in the Cosmos, NIC XI

July 19-23, 2010

Heidelberg, Germany

\footnotetext{
*Speaker.
} 


\section{Introduction}

From the point of view of element production, low- and intermediate-mass stars $\left(M<7-8 \mathrm{M}_{\odot}\right)$, during their Asymptotic Giant Branch (AGB) phase, are well known as the main site where nuclei heavier than iron are synthesized via slow neutron-capture processes [1]. These stars, however, are important manufacturing sites also for low and intermediate-mass elements, which come from H-burning. Indeed, presolar grains of AGB origins show unexpected carbon, nitrogen, oxygen and aluminum isotopic ratios far from the prescriptions of the canonical models of stellar evolution $[2,3,4]$. A similar puzzle exists for the photospheric CNO abundances of low mass stars, belonging the AGB and the previous Red Giant Branch (RGB) phase, which are not explicable in terms of the pure coupling of convection and nucleosynthesis $[5,6,7,8,9]$.

At the same time, low and intermediate mass stars play a crucial role in the galactic evolution of light elements, like $\mathrm{D},{ }^{3} \mathrm{He}$ and ${ }^{7} \mathrm{Li}$, mainly produced in the Big Bang but subsequently modified by stellar evolution. It has been argued that the same mechanism needed to destroy ${ }^{3} \mathrm{He}$ could provide also an explanation to the chemical anomalies of ${ }^{7} \mathrm{Li}$ and $\mathrm{CNO}$ isotopes observed in low-mass red giants [10]. Many studies have suggested that such anomalies can be accounted for by non-convective transport mechanisms (sometimes called "Cool Bottom Process" - CBP or "deep-mixing") linking the stellar envelope with the H-burning shell, during the red giant phases (RGB and AGB). Despite more than three decades of work there is not a clear consensus on the physical mechanism generating the mass transport (thermohaline diffusion [11], rotational shear $[12,13]$, magnetic buoyancy [14] being the most promising possibilities). Indications on the general properties of the phenomenon (velocity, effectiveness and depth) can however be obtained by fitting the observational constraints with physical or parametric models (see e.g. $[15,16]$ ). In this way one recognizes that $\mathrm{CNO}$ isotopes in evolved stars can be accounted for by various models; on the contrary, more stringent constraints are posed by ${ }^{26} \mathrm{Al}$-rich grains (requiring very deep mixing) or by Li-rich giants (requiring mixing mechanisms on time scales shorter than ${ }^{7} \mathrm{Be}$-decay).

A remarkable source of uncertainty in the nucleosynthesis that accompanies CBP episodes comes from the nuclear physic inputs, because H-burning takes place at Gamov-Peak energies of only a few tens of $\mathrm{keV}$, which fact leads to difficulties in experimental measurements of reaction rates. Most results present in the literature were obtained using reaction rates from either the [17] or the [18] compilations. Here we want to discuss the effects of recent upgrades in the nuclear physics inputs on p-capture nucleosynthesis during deep-mixing. For this scope we adopt the reaction rate recommendations from [19] (for nuclei with $\mathrm{A}<19$ ) and from [20] (for $19<\mathrm{A}<33$ ). In order to safely derive constraints on the construction of a physical model, we do not assume a priori any specific scenario for mixing, adopting the parametric approach by [16] and [21]. This was based on two free parameters; namely, the rate of mass transport $(\dot{M})$ and the temperature $\left(T_{P}\right)$ of the deepest zones reached by the circulation. In this paper we shall refer to this temperature through the logarithmic difference $\Delta \log T_{P}=\log T_{H}-\log T_{P}$, where $T_{H}$ is the temperature at which the maximum energy of the H-burning shell is released.

\section{Cool Bottom Process and H-shell Nucleosynthesis}

Panels a) and b) of Fig.1 present the comparison between two chemical compositions of the ra- 

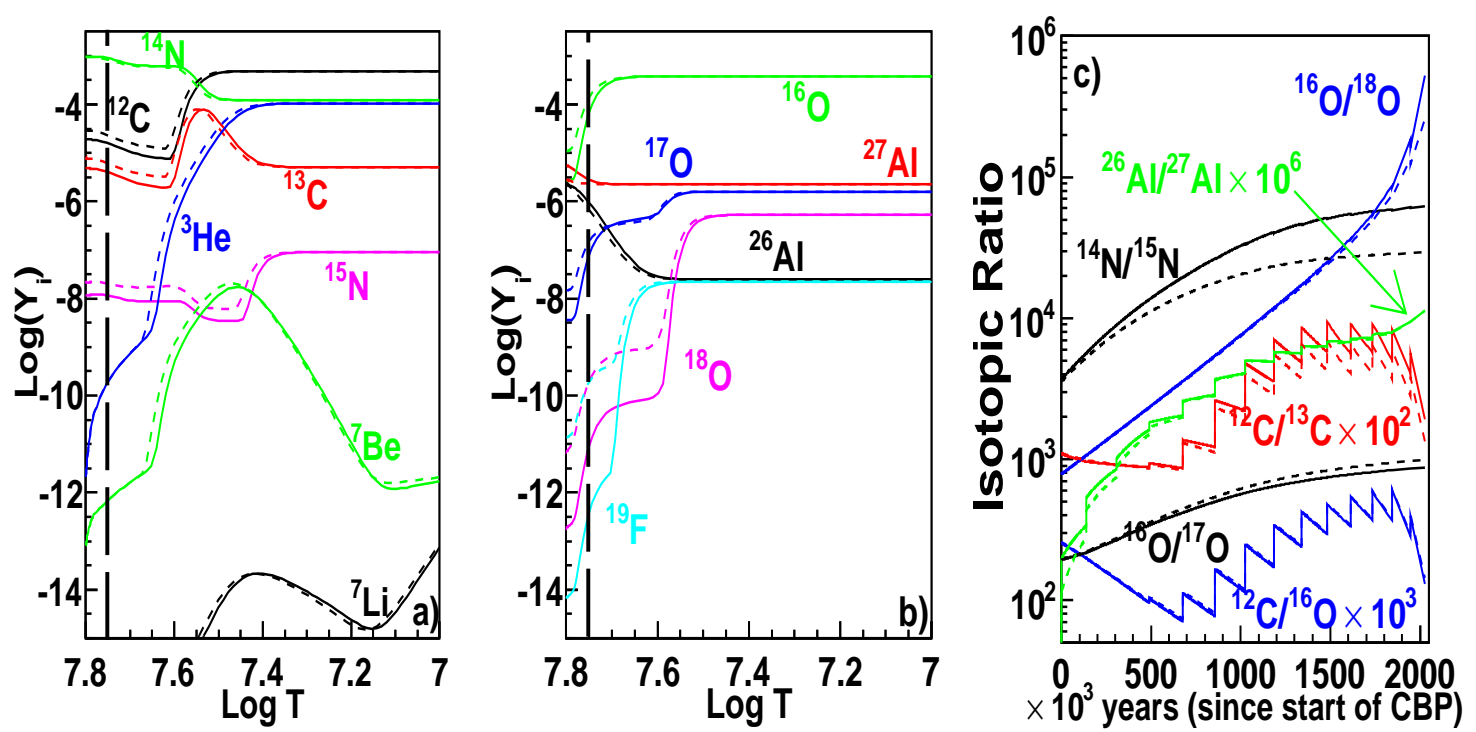

Figure 1: Panels a) and b): chemical composition of the radiative region below the convective envelope of a $2 M_{\odot}$, solar metallicity AGB star. Vertical dashed lines indicate the layers of maximum energy production $\left(T_{H}\right)$. Panel c): temporal evolution of isotopic ratios in the stellar envelope of the same model star of the previous panels. The abundances result from the occurrence of both deep mixing of partial H-burning ashes in the interpulse periods (with $\Delta \log T_{P}=0.1$ and $\dot{M}=3 \cdot 10^{-6} M_{\odot} / y r$ ) and of convective dredge-up of He-shell materials after thermal pulses. The comparison between solid lines (new rates) and dashed ones (NACRE) shows the effects of the nuclear input changes.

diative region, below the convective envelope, of a $2 M_{\odot}$, solar metallicity AGB star. The first (solid lines) results from the adoption of the new reaction rates; the second (dashes curves) has input data from the NACRE compilation. The stellar structures considered in our models were computed with the FRANEC code (see [22] for details). Among the changes introduced by [19], the most effective in inducing modifications of the nuclear yields is the reduction by a factor of two of the rate for the ${ }^{14} \mathrm{~N}(\mathrm{p}, \gamma){ }^{15} \mathrm{O}$. This being the slowest reaction of the $\mathrm{CNO}$ cycle, its reduced efficiency determines a shift of the H-burning shell to inner and warmers region of the star [22]. As a consequence, $\mathrm{T}_{H}$ increases by at least $6 \cdot 10^{6} \mathrm{~K}$ in red giants of $1.5-2 \mathrm{M}_{\odot}$, and all isotopes experience a more efficient proton capture process. Although the changes in the recommended values of reaction rates for the pp chain never exceed $20 \%$, the mentioned temperature change induces modifications also on light nuclei, like ${ }^{3} \mathrm{He},{ }^{7} \mathrm{Be}$ and ${ }^{7} \mathrm{Li}$. Individual nuclei also feel the effects of the specific rate changes relative to their own proton captures (see in particular the cases described in the next section). None of these upgrades are however as important as the one mentioned previously, for the ${ }^{14} \mathrm{~N}(\mathrm{p}, \gamma){ }^{15} \mathrm{O}$.

Finally, the ${ }^{26} \mathrm{Al} /{ }^{27} \mathrm{Al}$ ratio in $\mathrm{H}$-burning regions of $\mathrm{AGB}$ stars is almost unaltered, despite the new reaction rates from [20]. Fig. 1, Panel c, shows some relevant isotopic ratios in the envelope of our AGB model star, after the operation of the mixing mechanisms active in AGB phases. The abundances are produced assuming a contiunous process of deep mixing (with $\Delta \log T_{P}=0.1$ and $\left.\dot{M}=3 \cdot 10^{-6} M_{\odot} / y r\right)$ and adding its effects to those of the convective third dredge-up operating after each thermal pulse. It can be seen that clear shifts are present between the case using the NACRE 


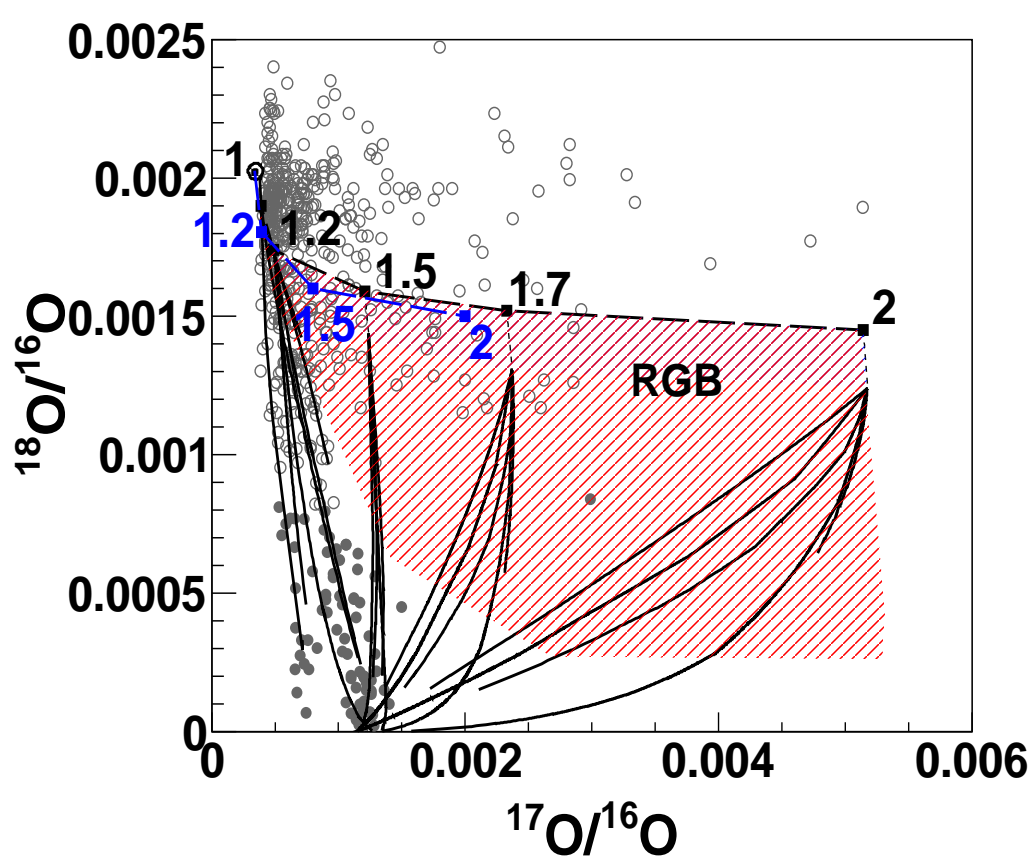

Figure 2: The dashed black curve represents the oxygen isotopic mix in the envelope of solar-metallicity stars at the first dredge-up. Full squares indicate the mass (from 1 to $2 M_{\odot}$ ) of the model stars. As a comparison,blue line and symbols show the composition for the masses $1.2,1.5$ and $2 M_{\odot}$ in the old scenario with NACRE rates. The shaded red area shows the range of values covered by a very efficient case of CBP on the RGB $\left(\Delta \log T_{P}=0.15\right.$ and $\left.\dot{M}=3 \times 10^{-7} M_{\odot} / y r\right)$. Continuous lines show model results for deep-mixing on the AGB, different lines refer to different mixing parameters. The occurrence of a moderate CBP during the RGB phase with $\Delta \log T_{P}=0.2$ and $\dot{M}=10^{-7} M_{\odot} / y r$ is also considered (short-dashed grey lines). The grey data points refer to measurements in presolar grains (from WUSTL Presolar Database http://presolar.wustl.edu/ $\sim$ pgd/). We plot those of group 1 (open circles) and of group 2 (filled circles). It is clear that $\mathrm{CBP}$ on the RGB, even operating with extreme efficiency, can not reproduce the data of group 2 grains. Models for $M \leq 1.7 M_{\odot}$ explain essentially all the data for ${ }^{18} \mathrm{O}$-poor grains. In particular, group 2 grains mainly derive from very low-mass stars (below $1.5 M_{\odot}$ ).

compilation and the one with the new rates. The main effects are on ${ }^{12} \mathrm{C} /{ }^{13} \mathrm{C},{ }^{14} \mathrm{~N} /{ }^{15} \mathrm{~N}$ and ${ }^{16} \mathrm{O} /{ }^{18} \mathrm{O}$.

\section{Crucial tests from presolar oxide grains}

Apparently from Fig.1 one might infer that very small changes occur for the oxygen isotopes. But Fig. 1 is partly misleading, because it shows the results of CBP calculations with the two sets of nuclear inputs, adopting common initial abundances. The main effects of the new rates is instead that of changing the initial AGB conditions sharply. This can be best appreciated by looking at the isotopic mix of oxygen in presolar oxide grains (from WUSTL Presolar Database http : //presolar.wustl.edu/ pgd/). Fig.2 shows the situation with both NACRE rates and those by [19]. The black line with numeric labels shows the isotopic composition at first dredge-up with the new choices. The blue line and its corresponding labels give, as a comparison, the previous 
situation with NACRE. The effects are huge, such that the situation is now a completely new one. The area of experimental data, at low ${ }^{17} \mathrm{O} /{ }^{16} \mathrm{O}$ now requires necessarily very low mass stars; presolar oxide grains turn out to descend from a very old population of AGB stars. Their small masses would prevent them from ever becoming $\mathrm{C}$-rich. The figure (and its caption) explains how deep mixing in the AGB stages is necessary for explaining the isotopic mix of the grains; it also reveals that virtually all the ${ }^{18} \mathrm{O}$-poor grains can be accounted for, without any forbidden area, contrary to previous conclusions [23]. The main cause for the strong revisions obtained in this case must again be ascribed to the ${ }^{14} \mathrm{~N}(\mathrm{p}, \gamma){ }^{15} \mathrm{O}$, as operating before the occurrence of the first dredge-up. Other important contributions derive form the improved physics of the FRANEC code and its revised opacities. By contrast, reactions rates on oxygen isotopes themselves have a comparatively small effect. For example, they change the equilibrium value of the ${ }^{17} \mathrm{O} /{ }^{16} \mathrm{O}$ ratio only by $15 \%$.

\section{References}

[1] M. Busso, R. Gallino, G. J. Wasserburg, ARA\&A, (1999), 37, 239.

[2] L.R. Nittler, C.M.O’D. Alexander, X. Gao, R.M. Walker,E. Zinner, Nucl. Phys. A, (1997), 621, 113.

[3] B.-G. Choi, G.R. Huss, G. J. Wasserburg, R. Gallino, Science, (1998), 282, 1284.

[4] R. S. Amari, L. R. Nittler, E. Zinner, R. Gallino, M. Lugaro, R. S. Lewis, ApJ, (2001), 546, 248.

[5] J.H. Harris, D. L. Lambert, D.L., V. V. Smith, ApJ, (1985) 299, 375.

[6] P. L. Cottrell, C. Sneden, C., A\&A, (1986) 161, 314.

[7] K. K. Gilroy, J. A. Brown, ApJ, (1991), 371, 578.

[8] M. D. Shetrone, C. Sneden, C. A. Pilachowski, PASP, (1993), 105, 686.

[9] R. Smiljanic, R. Gauderon, P. North, B. Barbuy, C. Charbonnel, N. Mowlavi, N., A\&A (2009), 502, 267.

[10] A. I. Boothroyd, I.-J. Sackmann, ApJ (1999) 510, 232.

[11] P. P. Eggleton, D. S. P. Dearborn, J. C. Lattanzio, Scice, (2006), 314, 1580.

[12] J.-P. Zahn, A\&A, (1992), 265, 115.

[13] C. Charbonnel, N. Lagarde, A\&A, (2010), 14432.

[14] M. Busso, G. J. Wasserburg, K. M. Nollett, A. Calandra, ApJ. (2007), 671, 802

[15] R. Guandalini, S. Palmerini, M. Busso, S. Uttenthaler, PASA, (2009) 26, 168.

[16] M. Busso, S. Palmerini, E. Maiorca, et al., ApJ, (2010), 717, L47.

[17] G. R. Caughlan, W. A. Fowler, Atomic Data and Nuclear Data Tables, (1988),40, 283

[18] C. Angulo, M. Arnould, M. Rayet, et al., Nucl. Phys. A, (1999), 656, 3.

[19] E. G. Adelberger, A. B. Balantekin, D. Bemmerer, et al., arXiv1004, (2010), 2318.

[20] C. Iliadis, R. Longland, A. E. Champagne, A. Coc, R. Fitzgerald, Nucl. Phys. A (2010), 841, 31.

[21] K. M. Nollett, M. Busso, M., G. J. Wasserburg, ApJ, (2003), 582, 1036.

[22] S. Cristallo, O. Straniero, R. Gallino, et al., ApJ, (2009), 696, 797.

[23] A. I. Boothroyd, I.-J. Sackmann,G. J. Wasserburg, ApJ, (1995), 442, L21. 